\title{
Light field moment imaging
}

\author{
Antony Orth ${ }^{1,2}$ and Kenneth B. Crozier ${ }^{1,3}$ \\ ${ }^{1}$ Harvard University School of Engineering and Applied Sciences, Cambridge, Massachusetts 02138, USA \\ ${ }^{2}$ e-mail: tonyorth@seas.harvard.edu \\ ${ }^{3}$ e-mail: kcrozier@seas.harvard.edu
}

Received April 10, 2013; revised June 25, 2013; accepted June 25, 2013; posted June 26, 2013 (Doc. ID 188491); published July 22, 2013

\begin{abstract}
We introduce a novel imaging technique called light field moment imaging (LMI) that uses the continuity equation to extract the first angular moments of a light field. We use these moments to construct perspective views of a scene. Examples of LMI in photography and microscopy are presented. (c) 2013 Optical Society of America OCIS codes: (110.1758) Computational imaging; (170.6900) Three-dimensional microscopy. http://dx.doi.org/10.1364/OL.38.002666
\end{abstract}

In the 2D representations of scenes captured by digital image sensors in conventional cameras and microscopes, significant depth information is discarded. Many applications, however, would benefit from an improved ability to record the 3D nature of the real world, and there are several techniques that aim to do so. In stereo imaging, the depths of points in a scene are determined from the disparity between two perspective views [1]. A method termed "shape from focus" extracts depth information from a focal stack of a scene, under the assumption that all objects are opaque [2]. In light field imaging, both the intensity and direction of rays impinging on the image sensor are recorded [3,4]. This enables images to be formed postcapture in which properties such as viewing perspective, aperture size, and focal plane position are varied. Light field imaging implementations, however, require significant modifications to conventional cameras and microscopes, e.g., the addition of a microlens array [3] or an absorbing mask [4].

We present a novel imaging technique in which the angular moments of light rays collected by an image sensor are determined. The angular moments are then used to reconstruct 3D perspective views of a scene. We call this light field moment imaging (LMI). Our method has the advantages that it only requires two images of a scene to be obtained at different focus distances and is implemented by using conventional cameras and microscopes. Unlike stereo imaging and shape from focus techniques, which can also produce perspective views [5,6], LMI makes no assumption as to the opacity of objects in the scene. LMI is therefore capable of producing 3D perspective views for partially absorbing objects, which are often encountered in biological microscopy. We present four examples of this method using common imaging systems and discuss further applications of LMI.

The light field function is a spatioangular description of irradiance in the ray optics regime and can take on a number of parameterizations [7]. In this work we use the parameterization $\bar{L}\left(x, y, z, \tan \theta_{X}, \tan \theta_{Y}\right)$ that represents the density of rays at a point $(x, y, z)$, propagating in the direction specified by angles $\theta_{X}$ and $\theta_{Y}$ [Fig. 1(a)]. Consider light rays propagating in air and define $\mathbf{V}=\left[\tan \theta_{X}, \tan \theta_{Y}, 1\right]$. Conservation of energy requires that the quantity $\bar{L} \mathbf{V}$ have zero divergence, leading to a continuity equation for the light field $\bar{L}$ :

$$
\begin{gathered}
\nabla \cdot(\bar{L} \mathbf{V})=0, \\
\partial \bar{L} / \partial z=-\nabla_{\perp} \cdot \bar{L}\left[\tan \theta_{X}, \tan \theta_{Y}\right] .
\end{gathered}
$$

The intensity recorded by an imaging system is related to the light field in object space via an integral over angular space: $I=\iint_{-\infty}^{\infty} \bar{L} R(\theta) \cos ^{3} \theta \mathrm{d} \tan \theta_{X} \mathrm{~d} \tan \theta_{Y}$, where $\theta$ is the angle between the ray and the image sensor normal and $R(\theta)$ captures the angular response of sensor pixels and illumination factors specific to the imaging system. In photography, for example, these illumination factors give rise to a $\cos ^{4} \theta$ dependence: $R(\theta) \cos ^{3} \theta=\cos ^{4} \theta[\underline{3}, \underline{8}]$. Typically, $R(\theta) \cos ^{3} \theta$ is absorbed into the angular dependence of $\bar{L}$ to create $L=\bar{L} R(\theta) \cos ^{3} \theta$, which we call the "recorded light field." This is the light field as seen at the image sensor plane in the camera, inclusive of vignetting and illumination factors. Following the light field literature, we will work with the recorded light field $L$ [3] .

Multiplying Eq. (2) by $R(\theta) \cos ^{3} \theta$ and integrating over angular space yields a Poisson equation:

$$
\frac{\partial I(x, y ; z)}{\partial z}=-\nabla_{\perp}^{2} U(x, y ; z) .
$$

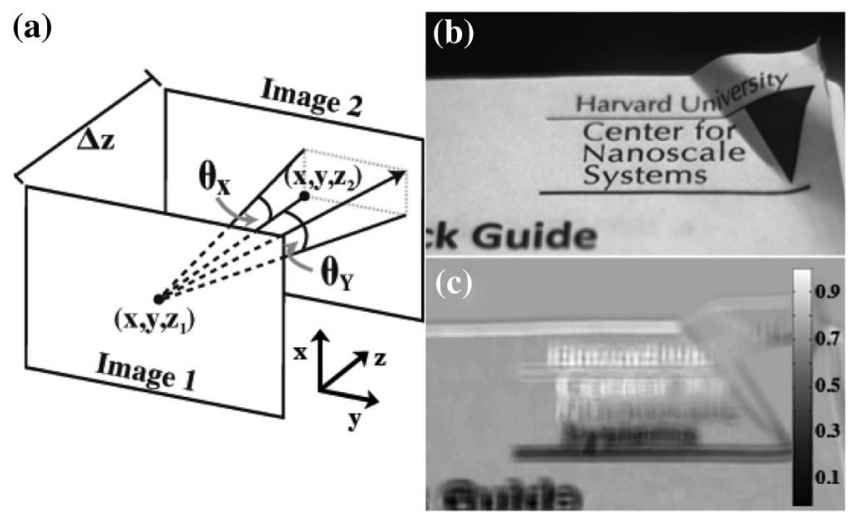

Fig. 1. (a) Schematic of the light field parameterization used. $\theta_{X}$ and $\theta_{Y}$ are defined by the projections of a ray onto the $x z$ and $y z$ planes, respectively. (b) A photograph of a bent sheet of paper. A video showing two images at different focal planes, followed by an animation of the viewpoint traveling horizontally is shown as Media 1. (c) Normalized depth map of the sheet with gray-scale bar indicating depth. Normalized depth ranges from 0 (closest to camera) to 1 (farthest from camera). 
Here, we have defined a scalar potential $U(x, y ; z)$ by $\nabla U(x, y ; z)=I(x, y ; z) \mathbf{M}(x, y ; z)$, where $\mathbf{M}=[s(x, y ; z)$, $t(x, y ; z)]$ is a vector containing the normalized first moments of the light field $L$ over its angular coordinates:

$$
[s, t]=\frac{\iint_{-\infty}^{\infty} L\left[\tan \theta_{X}, \tan \theta_{Y}\right] \mathrm{d} \tan \theta_{X} \mathrm{~d} \tan \theta_{Y}}{\int_{-\infty}^{\infty} L d \tan \theta_{X} \mathrm{~d} \tan \theta_{Y}} .
$$

Equation (3) is solved in Fourier space, and $\mathbf{M}$ is calculated via its relationship to $U(x, y ; z)$ [9]. We implement this solution in MATLAB by applying a filter $H$ to the approximate derivative calculated by taking the difference between the two input images $I_{1}=I\left(x, y ; z_{1}\right)$ and $I_{2}=I\left(x, y ; z_{2}\right)$ focused at different planes:

$$
\begin{gathered}
H\left(f_{x}, f_{y}\right)=\left[-4 \pi^{2}\left(f_{x}^{2}+f_{y}^{2}\right)\right]^{-1} \quad \text { for }\left(f_{x}^{2}+f_{y}^{2}\right) \neq 0, \\
H\left(f_{x}, f_{y}\right)=1 \quad \text { for }\left(f_{x}^{2}+f_{y}^{2}\right)=0, \\
U=\operatorname{IFT}\left[H \times \operatorname{FT}\left\{\left(I_{1}-I_{2}\right) / \Delta z\right\}\right],
\end{gathered}
$$

where FT and IFT denote the Fourier transform and its inverse, respectively; $f_{x}$ and $f_{y}$ are the spatial frequencies in the $x$ - and $y$-directions, respectively, and $\Delta z=z_{1}-z_{2}$. The moment vector field $\mathbf{M}$ is then calculated by applying the MATLAB gradient function to $U$ and dividing the result by $\left(I_{1}+I_{2}\right) / 2$. In cases where the defocus distance $\Delta z$ between images is not known by the user, the light field moments are found to be a multiplicative constant; i.e., we do now know their absolute values. This has no effect on the $3 \mathrm{D}$ visualizations shown in this work, however.

We construct an approximation to the light field of the scene at $z_{1}$ by assuming that the angular distribution of rays is Gaussian with mean $[s(x, y), t(x, y)]$ :

$$
\begin{aligned}
L\left(x, y, \tan \theta_{X}, \tan \theta_{Y}\right)= & I_{2}(x, y) \\
& \times \exp \left\{-\left[\tan \theta_{X}-s(x, y)\right]^{2} / \sigma^{2}\right. \\
& \left.-\left[\tan \theta_{Y}-t(x, y)\right]^{2} / \sigma^{2}\right\} .
\end{aligned}
$$

We empirically set $\sigma^{2}=\mathrm{NA}^{2}$ and have dropped $z_{1}$ for convenience. We have found that this $\sigma$ value gives a good perspective shifting effect. Further investigation is needed to understand and improve on this Gaussian assumption. This will be the subject of further research. The Gaussian assumption enables the reconstruction of a scene as viewed at different perspectives by choosing $2 \mathrm{D}$ slices of the light field in Eq. (6) at a given $\left(\tan \theta_{X}, \tan \theta_{Y}\right)$ coordinate [3].

As a first example of this technique, we photograph a bent sheet of paper with a gray-scale digital camera (Basler acA2000-340 km) equipped with a single lens reflex camera lens (50 mm focal length, $f / 1.4$ ). A pair of images is recorded at slightly different focus distances. Using the difference between these images to approximate the left-hand side of Eq. (3), we solve the Poisson equation and construct $L$ as in Eq. (6). Finally, we extract perspective views of the sheet at different positions along the horizontal axis. Each perspective shifted image in the animation is normalized such that its highest intensity pixel has a value of 1 . Parallax manifests itself as apparent movement of features when the viewpoint is changed. When viewing an animation where the viewpoint travels horizontally, as in Fig. 1(b) (Media 1), our brain efficiently processes the temporal sequence and can easily recognize depth within the image [10]. Here, the sheet is slanted such that the top of the paper is farther away from the camera than the bottom. One can also use the light field created with Eq. (6) in order to calculate a depth map. Depth maps based on disparity assign a depth value to each pixel based on the amount displacement of a feature between images taken from different viewpoints [1]. We use a plenoptic depth-mapping algorithm to produce the depth map depicted in Fig. 1(c) [11]. As is expected, the depth map indicates that the bottom of the sheet is closer to the camera than the top. Areas of the image without features-such as blank areas of the page-do not return proper depth values. This is typical of depth mapping algorithms that rely on disparity [11].

Viewpoint synthesis can also be performed with images acquired using a microscope. Optical microscopes are particularly well suited to recording focal stacks because they are equipped with a stage that translates along the optical axis. We image a tissue microarray section (US Biomax BLSC 1501) by using an optical microscope operated in transmission mode, with a $10 \times 0.3 \mathrm{NA}$ objective and fitted with a color digital camera; a micrograph of the tissue sample is shown in Fig. 2(a). We proceed with the same image-processing scheme as outlined above, for each color channel separately. The tissue section has been folded upon itself such that it has an appreciable 3D structure. We used two input images focused at

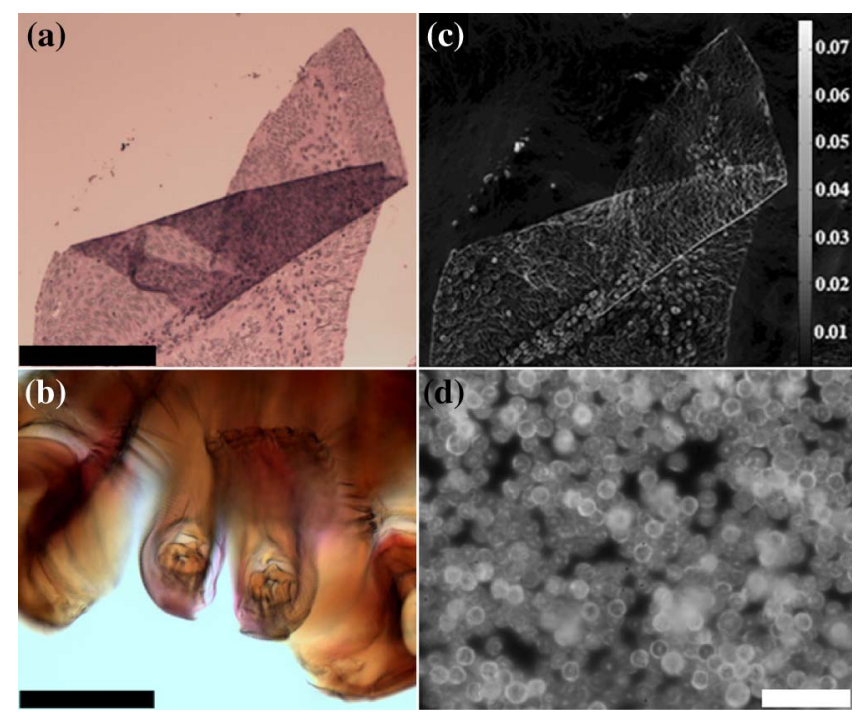

Fig. 2. (a) Micrograph of a folded tissue slice. Scale bar is $200 \mu \mathrm{m}$. The two input images and a perspective-shifting animation of the tissue slice are shown as Media 2. (b) Micrograph of the head of a wood tick. Scale bar is $200 \mu \mathrm{m}$. The two input images and a perspective-shifting animation of the wood tick head are shown as Media 3. (c) Gray-scale plot of the magnitude of the moment vector field $\mathbf{M}$ for the tissue sample in (a). The gray-scale bar shows the dimensionless value of $|\mathbf{M}|$. (d) Micrograph of a pile of $5 \mu \mathrm{m}$ fluorescent beads. Scale bar is $25 \mu \mathrm{m}$. The two input images and a perspective-shifting animation of this sample are shown as Media 4. 
planes separated by $\Delta z=6 \mu \mathrm{m}$ in object space. An animation of the tissue section as seen from a viewpoint traveling along the vertical axis is shown in Fig. 2(a) (Media 2). To further showcase the ability of LMI to create perspective shifted images of complex translucent samples, we imaged the head of a wood tick (Dermacentor variabilis, Carolina Biological Supply) at two planes separated by $\Delta z=5 \mu \mathrm{m}$. We use the same microscope and objective as above. A micrograph of the tick head and an animation showing the reconstructed perspective views are shown as Fig. 2(b) (Media 3). Reconstruction of perspective views using a depth map would be impossible in this partially transparent sample because a single depth value cannot be assigned to a given position in the image [].

In addition to perspective shifting, one can construct an image similar to a dark field micrograph by plotting the magnitude of $\mathbf{M}$, as shown in Fig. 2(b). Because the microscope uses Kohler illumination, which is symmetric about the optical axis, we expect that $|\mathbf{M}|=0$ in the absence of scatterers. Indeed, this is observed away from the tissue section. However, small features such as cells tend to scatter strongly and anisotropically. Therefore these localized scattering features give rise to large $|\mathbf{M}|$ values and appear bright in Fig. 2(b). The maximum $|\mathbf{M}|$ value is limited by the NA of the microscope objective. In Fig. 2(b), $|\mathbf{M}|<0.08$, which is consistent with our expectation that $|\mathbf{M}|<\tan \left[\sin ^{-1}(0.3)\right]=0.31$.

LMI can also be used in fluorescence microscopy. To demonstrate this, we image a pile of $5 \mu \mathrm{m}$ fluorescent beads (Invitrogen) with a $50 \times 0.55 \mathrm{NA}$ microscope objective. A $532 \mathrm{~nm}$ laser is used to excite the fluorescence, and a long-pass filter is placed in front of the image sensor (Basler acA2000-340 km) so that it only collects the fluorescent emission from the beads. A micrograph of the sample along with a perspective shifting animation is shown as Fig. 2(d) (Media 4). Again, parallax is apparent in the animation.

Noise amplification at low spatial frequencies occurs because of the Fourier filter [Eqs. (5a) and (5b)] that is used to solve the Poisson equation. If the two images used are too closely spaced, this noise can dominate the solution to the Poisson equation [12]. This problem could be mitigated in a number of ways, for example by capturing more images to cut down on noise [9] or by blending together perspective images constructed by using pair of images of different axial separations [12]. This problem has been studied extensively in the phase imaging literature $[9,12]$; we leave the implementation of noise reduction techniques to future work. In Fig. 2(a) (Media 2), noise manifests itself as coloration in the perspective images. This is because of noisy low spatial frequency modes in the perspective images that vary between color channels. The result is the emergence of patches of false colors, which are especially prominent in areas dominated by low spatial frequency features.

We have shown how a continuity equation for light fields can be exploited to calculate the first angular moments of a light using a pair of input images. With these moments, we can reconstruct perspective-shifted views of the original scene under the empirical assumption that the angular distribution of the light field is Gaussian. Depth maps and dark-field-like microscope images can also be extracted with LMI. Our approach is versatile, as we have demonstrated it on three different imaging systems ranging from photographic cameras to microscopes. LMI enables convenient 3D perspective imaging in systems where light field sensors might be impractical such as high-resolution microscopes and mobile phone and infrared cameras. In microscopy, LMI could be an alternative to confocal fluorescence microscopy, with the advantage that the excitation dose is lower. In cinematography, LMI might be an efficient way to capture next generation 3D media for light field displays [13]. We expect that this technique will be applicable to more exotic focused imaging systems that obey the ray approximation, such as transmission electron microscopes.

\section{References}

1. T. Kanade, A. Yoshida, K. Oda, H. Kano, and M. Tanaka, in IEEE Computer Society Conference on Computer Vision and Pattern Recognition, 1996. Proceedings CVPR'96, 1996 (IEEE, 1996), p. 196.

2. S. K. Nayar and Y. Nakagawa, in 1990 IEEE International Conference on Robotics and Automation, 1990. Proceedings (IEEE, 1990), p. 218.

3. R. Ng, ACM Trans. Graph. 24, 735 (2005).

4. A. Veeraraghavan, R. Raskar, A. Agrawal, A. Mohan, and J. Tumblin, ACM Trans. Graph. 26, 69 (2007).

5. D. Scharstein and R. Szeliski, in IEEE Computer Society Conference on Computer Vision and Pattern Recognition, 2003. Proceedings, Vol. 1 (IEEE, 2003), p. 195.

6. M. Noguchi and S. K. Nayar, in Proceedings of the 12th IAPR International Conference on Pattern Recognition, 1994, Vol. 1 (IEEE, 1994), p. 147.

7. M. Levoy, Z. Zhang, and I. McDowall, J. Microsc. 235, 144 (2009).

8. F. A. Jenkins and H. A. White, Fundamentals of Optics, 3rd ed. (McGraw-Hill, 1957).

9. L. Waller, L. Tian, and G. Barbastathis, Opt. Express 18, 12552 (2010).

10. J. W. Nadler, D. E. Angelaki, and G. C. DeAngelis, Nature 452, 642 (2008)

11. E. H. Adelson and J. Y. Wang, IEEE Trans. Pattern Anal. Mach. Intell. 14, 99 (1992).

12. D. Paganin, D. Barty, P. J. McMahon, and K. A. Nugent, J. Microsc. 214, 51 (2004).

13. D. Fattal, Z. Peng, T. Tran, S. Vo, M. Fiorentino, J. Brug, and R. Beausoleil, Nature 495, 348 (2013). 